\title{
Quelques Réflexions sur l'Assurance Maladie
}

\author{
par Ruy de Carvalho*
}

\section{Assurance maladie privée; Etats-Unis et Europe}

Le panel organisé à Zurich, par l'Association de Genève, l'année passée, à l'occasion de la 13e Assemblée Générale, a traité l'évolution de l'assurance Responsabilité Civile sous une double perspective: celle des Etats-Unis et celle de l'Europe.

Dans le panel de cette année, sur la fonction complémentaire de l'assurance maladie privée par rapport aux systèmes de sécurité sociale, on compare de nouveau les réalités de ces deux marchés.

C'est une décision très opportune, non seulement parce que les comparaisons de différentes expériences sont toujours enrichissantes, mais aussi parce que dans certains champs d'activité, comme celui en question, le présent aux Etats-Unis devient souvent le proche avenir de l'Europe. On peut donc féliciter l'Association de Genève de cette initiative.

Bien que cette première partie du panel soit consacrée à la situation présente, il est difficile d'en parler sans réfléchir sur le futur immédiat de l'assurance maladie. Et il faut se contenter de ce futur immédiat parce que, à l'heure actuelle, avec un environnement en constante mutation, il serait trop risqué de vouloir penser à plus long terme.

D'autre part, même considérant que la situation aux Etats-Unis est parfois très distincte de celle de l'Europe, il est utile de l'examiner, puisque cela peut éviter certaines erreurs qui peuvent compromettre l'avenir de cette branche.

En même temps, l'expérience américaine peut aider à trouver des solutions pour les problèmes récents concernant cette assurance, tels que:

- l'augmentation explosive des coûts;

- les difficultés de leur contrôle;

- le besoin de garantir la qualité des soins de santé, malgré les charges que cela représente;

- le manque de rentabilité de certaines HMO;

* Président, Associaçao Portuguesa de Seguradores; Texte présenté à l'Assemblée Générale de l'Association de Genève, Berlin, 16 juin, 1987. 
bien qu'il faille tenir compte de la pénétration très différente de l'assurance maladie privée dans les deux marchés en question (environ $65 \%$ aux Etats-Unis et $15 \%$ en Europe), ce qui découle naturellement des systèmes sociaux en vigueur et de la philosophie qui inspire les options prises par chacun des gouvernements dans la matière. Il ne faut pas oublier que l'encaissement de primes d'assurance privée en Europe ne représente plus que 12 à $15 \%$ du total de l'assurance maladie obligatoire.

\section{L'évolution des soins de santé}

Avant d'entrer dans la problématique même de l'assurance maladie, examinons très rapidement quelques modifications qui se sont opérées dans le domaine des soins de santé.

Concentrons-nous, pour le moment, sur certains aspects qui peuvent contribuer à l'amélioration de la situation, et qui sont liés:

- à l'être humain;

- à l'environnement;

- aux moyens extérieurs applicables.

En ce qui concerne le perfectionnement de l'être humain, il convient de rappeler le mouvement initié en Californie, il y a une bonne douzaine d'années, selon lequel la préoccupation centrale ne serait plus la maladie mais la préservation de la santé ${ }^{1}$.

Dans cette perspective, la place d'honneur vient donc à la prévention et c'est la raison pour laquelle, pendant les années 70 s'est tellement developpé l'intérêt porté aux régimes alimentaires et à la nutrition, ainsi qu'à la protection contre les effets nocifs de l'environnement sur la santé.

C'est alors que le concept de "santé globale" (wholistic health) est devenu de plus en plus populaire.

Et si "bien-être" et "santé globale" ont parfois été confondus avec des traitements expérimentaux ou avec l'usage de médecines alternatives, cela ne correspond pas du tout à la réalité, puisque le concept de "santé globale" signifie très simplement soigner, dans son ensemble, le corps, les émotions et l'esprit.

En effet, un programme-type de "santé globale" inclut:

- la pratique régulière d'exercices physiques;

- l'abstention de fumer;

- une nutrition salutaire (peu de graisse, sel, sucre et alcool);

- du repos adéquat;

- le contrôle de l'état de tension (stress).

La communauté des affaires a apporté son concours à cette tendance, née en Californie, notamment à travers la constitution de centres de santé (health centers), d'amaigrissement et de prévention.

En ce qui concerne l'environnement, sa conservation et l'influence que cela peut avoir sur notre santé, on l'a commenté tellement souvent qu'on peut, à ce stade, ne rien ajouter.

\footnotetext{
${ }^{1}$ Megatrends - John Naisbitt - Warner Books 1982.
} 
Quant à l'application d' agents extérieurs pour améliorer la santé, tels que les médicaments, les soins médicaux et la chirurgie, on peut citer:

- l'organisation des institutions de santé;

- l'usage de nouvelles technologies sophistiquées;

- les aspects sociaux et pathologiques.

Pour ce qui est des institutions de santé, on assiste à une popularité grandissante des organisations locales privées gérées par les propres intéressés (self-help) par rapport aux organisations institutionnelles. Et ceci comme résultat d'une certaine perte de confiance dans l'institution médicale (suite à son incapacité de guérison de certaines maladies), une tendance à des solutions plus flexibles et personnalisées, ainsi que le souci de voir monter continuellement les prix des soins de santé. C'est un peu dans cet esprit que les HMO se sont developpés aux Etats-Unis.

L'usage de technologies plus sophistiquées a permis:

- de nouvelles possibilités de diagnostic et de traitement;

- la réalisation plus fréquente de soins et de tests à domicile, moins chers que les solutions traditionnelles;

mais a eu aussi comme conséquences négatives:

- des rapports souvent peu satisfaisants entre les coûts et leur efficacité (cost-effectiveness);

- des tendances au surtraitement (overtreatment).

Finalement, quant aux aspects pathologiques et sociaux, il faut souligner l'importance de certaines nouvelles maladies qui se développent, ou simplement qui sont diagnostiquées grâce à l'usage de moyens plus sophistiqués ou à des progès réalisés dans le domaine de l'investigation.

Le cas le plus connu actuellement et qui a plus d'impact sur l'humanité et aussi sur certaines modalités d'assurance est naturellement celui du SIDA.

Dans ce cas, il y a non seulement le problème crucial de la découverte des moyens de guérison et de la production d'un vaccin efficace, qui pourrait empêcher la propagation de cette maladie à l'allure actuelle, mais aussi les problèmes d'ordre social et de responsabilité civile, qui entravent les travaux d'investigation. En effet, les tentatives d'investigation se heurtent, dans certains cas, à des contraintes qui proviennent de la crainte de procès de responsabilisation des investigateurs et des laboratoires qui pourraient produire ce vaccin, étant donné que l'être humain paraît, au moins à l'heure actuelle, en être le seul champ d'expérimentation et que cela peut poser des problèmes très délicats.

Mais à part le SIDA, quelles sont les nouvelles maladies qui peuvent apparaître à l'avenir? Et quelles conséquences peuvent-elles entraîner pour les assureurs, surtout parce que, tel le cas du SIDA, il se peut que ces nouvelles maladies aient une période plus ou moins longue d'incubation, ce qui cause des difficultés au dépistage et, en ce qui concerne l'assurance privée, le calcul correct des primes correspondantes? Ce sont des questions auxquelles il serait bien utile de pouvoir trouver une réponse. 
Voici une très brève panoramique de quelques problèmes que pose l'évolution des maladies, des soins de santé et des moyens d'assistance disponibles.

Malgré cette description beaucoup trop simpliste, il n'est pas difficile d'imaginer quelles conséquences ladite évolution peut avoir sur le comportement des marchés de l'assurance qui continuent à être, malgré tous les efforts, très différents d'un pays à l'autre, même au sein de la Communauté européenne.

\section{Prévoyance privée et assurance}

La répartition de notre temps de vie entre l'éducation, le travail et la retraite se fait, de moins en moins, selon le paradigme traditionnel où chacune de ces étapes était bien compartimentée et échelonnée dans le temps.

Comme dans d'autres domaines, les modifications dans les habitudes entraînent la nécessité d'une croissante flexibilité des solutions et chacune des phases indiquées s'interpénètrent de plus en plus.

Les changements les plus accentués vont certainement encore apparaître dans la nature, les moyens et le temps consacré à la formation active et passive, ainsi que dans les habitudes, le genre d'activité et le pouvoir économique des retraités.

En ce qui concerne le financement des soins de santé, les alternatives possibles sont:

- les systèmes de sécurité sociale;

- la prévoyance privée, en utilisant l'assurance ou le recours direct aux organisations spécialisées dans les soins de santé.

La rigidité des systèmes d'assurances sociales, qui se sont développés surtout à partir du début du siècle, ne permet plus de répondre aux tendances et besoins actuels, à part les difficultés de financement que ces systèmes connaissent actuellement dans la grande généralité des pays, pour des raisons bien connues, notamment d'ordre démographique et de niveau d'emploi.

C'est dans ce cadre que les systèmes privés complémentaires de la sécurité sociale gagnent très vite une place majeure et que les théories des deuxième et troisième piliers et du retour à la capitalisation, ont chaque fois plus d'adeptes.

Et dans ce domaine de la prévoyance d'entreprise ou individuelle, l'assurance privée est un instrument privilégié, notamment par sa flexibilité et sa capacité de garantir une couverture globale.

Etant assez flexible, quant aux couvertures assurées, à son propre coût et à sa répartition entre l'entité patronale et les bénéficiaires, l'assurance s'adapte donc plus facilement à l'évolution de la capacité économique des parties contractantes et aux changements d'emploi et d'habitudes de vie, chaque fois plus fréquents.

L'assurance est, en même temps, on l'a déjà mentionné, une bonne solution sous l'aspect de sa globalité. Et si l'on se place, pour un instant, sous la perspective plus vaste des différentes modalités d'assurance, et non seulement de l'assurance maladie, on constate qu'il existe toute une gamme de produits qui permettent de garantir notamment: 
- l'assistance en cas d'accident, de travail ou autres, de déplacements en service ou à titre privé, de maladie et d'incapacité temporaire ou définitive;

- le complément de retraite dûment adapté aux circonstances, souvent changeantes, de celle-ci;

- la survie des descendants ou du conjoint, surtout en cas de mort prématurée.

L'importance c'est de trouver le "package" le plus ajusté à chaque cas ou à chaque entreprise, ce qui est un travail de coopération entre assureurs et assurés.

Et dès qu'on pense à la protection de l'individu, il ne faut pas négliger la tendance actuelle, très marquée, d'exigences accrues dans le domaine de la responsabilité civile et de sa couverture par l'assurance.

Si l'on pense à l'évolution aux Etats-Unis, et même en Europe, de quelques cas concernant la RC Environnement et la RC Produits, ainsi qu'à la possibilité de rembourser certains frais de soins médicaux par des polices couvrant la Responsabilité Civile, on s'aperçoit d'une interpénétration croissante des différentes classes d'assurances, dont on doit aussi tenir compte lorsqu'on prépare un plan global de couverture.

Mais l'assurance ne peut pas être la solution miraculeuse pour tous les problèmes concernant l'assistance à la santé car elle a aussi ses difficultés.

Avant d'en énumérer quelques-unes, examinons rapidement un aspect préalable qui est celui des perspectives d'exploitation. On à déjà fait allusion, quant aux soins de santé, que deux aspect distincts doivent être considérés:

- les mesures préventives;

- l'assistance en cas de maladie, visant avant tout et, dans les cas où il est possible, la guérison.

Les principaux intervenants dans ce processus sont donc:

- le propre bénéficiaire des soins de santé ou de mesures de prévention de la maladie;

- les services médicaux et hospitaliers;

- les fournisseurs de moyens de diagnostic, thérapie et prévention, ainsi que ceux des médicaments;

- les chercheurs;

- les organismes de fiancement du système.

Pour que les difficultés financières de la sécurité sociale ne soient pas, au moins partiellement, transférées aux sociétés d'assurance, il faut:

- inciter à la modération;

- limiter les exigences aux prestations effectivement dues et cliniquement utiles;

- contrôler les prix, tout en gardant néanmoins une qualité adéquate. Cet aspect est parmi les plus décisifs pour l'avenir de l'assurance maladie;

- stimuler la médecine préventive et son utilisation par le public. 
Si l'on ne tient pas compte de ces préoccupations et si l'on ne cherche pas à les résoudre, il sera extrêmement difficile d'aboutir à une exploitation rentable de l'assurance maladie privée, qui se débat, en toutes circonstances, avec les problèmes suivants:

- les conséquences négatives d'une évolution démographique, selon laquelle le pourcentage de personnes âgées s'accroît et provoque une augmentation très sensible des soins de santé et par conséquent des coûts;

- les changements d'ordre social, moral et économique qui entraînent des modifications sensibles dans la notion et le rôle de l'agrégat familial et dans les possibilités d'entraide des membres de la famille;

- le manque de statistiques fiables, suffisament significatives et disponibles à temps d'en retirer des conclusions et de prendre des décisions stratégiques pour réagir, avec efficacité, aux changements d'environnement;

- l'évolution et le manque d'harmonisation entre les régimes fiscaux applicables à ce genre d'assurances;

- les traditions qui veulent, qu'en Europe, seulement une faible partie de ces risques soit placée auprès des assureurs, provoquant une certaine anti-sélection;

- le manque de contrôle sur les prix d'assistance à la santé, allié à une concurrence parfois très vive entre assureurs;

- des difficultés pour assurer les soins médicaux en cas de maladies de longue durée (longterm care), pour lesquelles la plupart des pays ne possèdent pas encore de solutions institutionnelles suffisamment efficaces.

Pour terminer ces brèves réflexions sur l'assurance et son rôle complémentaire des systèmes de sécurité sociale, je voudrais insister sur deux questions: l'importance de la qualité du service rendu et l'utilisation croissante des plans flexibles d'assurance maladie (multioption plans). Quant à la première, c'est plutôt-là et moins dans le facteur prix de l'assurance, que doivent se concentrer les efforts des assureurs.

Quant à la deuxième question, les plans qui permettront aux assurés de choisir la meilleure combinaison entre les systèmes de santé, en associant aux systèmes basés sur les indemnités des systèmes de management des soins (managed care), seront l'instrument privilégié de l'assurance maladie.

Ces deux questions seront donc, à l'avenir, parmi les préoccupations prioritaires des assureurs européens les plus compétitifs et aussi les plus performants dans ce domaine.

\section{L'avenir en Europe}

Au début de cet exposé, on a considéré que le présent aux Etats-Unis devient souvent le proche avenir en Europe.

Si l'on examine la situation actuelle dans ces deux régions, on constate aux Etats-Unis une influence majeure de l'initative privée dans ce domaine, tandis qu'en Europe le poids des systèmes étatisés est encore considérable. 
La dimension des entreprises où se pose le problème d'une assistance à travers le deuxième pilier est aussi très différente. Un système bien adapté aux besoins des PME est indispensable en Europe.

Les réalités des divers états d'Amérique du Nord sont certainement différents de celles qui marquent, du point de vue économique, social, culturel et politique, les pays européens.

Les rapprochements et les harmonisations seront certainement plus longues et difficiles en Europe.

L'utilisation plus fréquente aux Etats-Unis, de programmes multi-options s'appuie sur des organisations telles que les HMO et les PPO, ce qui pose quelques réserves sur son applicabilité, telle quelle, en Europe. Les organisations privées du type "self-help" n'étant pas, du moins pour le moment, répandues en Europe, cela établit de nouveau une différence entre les réalités des deux marchés.

Les modèles américains ne peuvent donc servir que de modèles de référence, lorsqu'on réfléchit sur le développement futur de l'assurance maladie privée européenne.

Mais on peut certainement en retirer des suggestions valables, et s'en servir pour éviter certaines erreurs qui peuvent entraîner de grosses pertes dans nos systèmes privés d'assistance à la santé, lesquels vont indiscutablement devoir compléter les systèmes de sécurité sociale.

L'importance c'est d'arriver à une coopération au lieu d'une confrontation, tout en sauvegardant la rentabilité du système privé.

Un transfert de pertes d'un secteur à l'autre n'est pas concevable, mais le développement des deuxième et troisième piliers, aussi dans ce domaine, est une réalité visible et très souhaitable.

Il est aussi possible, qu'en Europe, des réseaux privés d'assistance se développent, en nombre limité mais avec des dimensions et capacités économiques et financières significatives, sans qu'on puisse oublier, toutefois, les caractéristiques propres aux différents pays européens où ces réseaux pourraient opérer.

Un changement est en cours et cette certitude doit mobiliser toute notre attention, notre détermination et notre capacité de réponse. Elles ne nous manqueront pas, j'en suis sûr, le moment venu. 